\title{
ON WEAK CHROMATIC POLYNOMIALS OF MIXED GRAPHS
}

\author{
MATTHIAS BECK, DANIEL BLADO, JOSEPH CRAWFORD, TAÏNA JEAN-LOUIS, AND MICHAEL YOUNG
}

\begin{abstract}
A mixed graph is a graph with directed edges, called arcs, and undirected edges. A $k$-coloring of the vertices is proper if colors from $\{1,2, \ldots, k\}$ are assigned to each vertex such that $u$ and $v$ have different colors if $u v$ is an edge, and the color of $u$ is less than or equal to (resp. strictly less than) the color of $v$ if $u v$ is an arc. The weak (resp. strong) chromatic polynomial of a mixed graph counts the number of proper $k$-colorings. Using order polynomials of partially ordered sets, we establish a reciprocity theorem for weak chromatic polynomials giving interpretations of evaluations at negative integers.
\end{abstract}

\section{INTRODUCTION}

A mixed graph $G=(V, E, A)$ consists of a set of vertices, $V=V(G)$, a set of undirected edges, $E=E(G)$, and a set of directed edges, $A=A(G)$. For convenience, the elements of $E$ will be called edges and the elements of $A$ will be called arcs. Given adjacent vertices $u, v \in V$, an edge will be denoted by $u v$ and an arc will be denoted by $\overrightarrow{u v}$.

A $k$-coloring of a mixed graph $G$ is a mapping $c: V \rightarrow[k]$, where $[k]:=\{1,2, \ldots, k\}$. A weak (resp. strong) proper $k$-coloring of $G$ is a $k$-coloring such that

$$
c(u) \neq c(v) \text { if } u v \in E \quad \text { and } \quad c(u) \leq c(v) \quad(\text { resp. } c(u)<c(v)) \text { if } \overrightarrow{u v} \in A .
$$

The weak (resp. strong) chromatic polynomial, denoted by $\chi_{G}(k)$ (resp. $\widehat{\chi}_{G}(k)$ ), is the number of weak (resp. strong) proper $k$-colorings of $G$. It is well known (see, e.g., [4, 5]) that these counting functions are indeed polynomials in $k$. Coloring problems in mixed graphs have various applications, for example in scheduling problems in which one has both disjunctive and precedence constraints (see, e.g., [2, 3, 6]).

An orientation of a mixed graph $G$ is obtained by orienting the edges of $G$, i.e., assigning one of $u$ and $v$ to be the head/tail of the edge $u v \in E$; if $v$ is the head we use the notation $u \rightarrow v$. (An arc $\overrightarrow{u v}$, for which we also use the notation $u \rightarrow v$, cannot be re-oriented.) An orientation of a mixed graph is acyclic if it does not contain any directed cycles. A mixed graph is acyclic if all of its possible orientations are acyclic. A coloring $c$ and an orientation of $G$ are compatible if for every $u \rightarrow v$ in the orientation, $c(u) \leq c(v)$.

A famous theorem of Stanley says that, for any graph $G=(V, E, \varnothing)$ and positive integer $k$, $(-1)^{|V|} \chi_{G}(-k)$ enumerates the pairs of $k$-colorings and compatible acyclic orientations of $G$ and, in particular, $(-1)^{|V|} \chi_{G}(-1)$ equals the number of acyclic orientations of $G$ [8]; this is an example of

Date: 11 June 2013.

2000 Mathematics Subject Classification. Primary 05C15; Secondary 05A15, 06A07.

Key words and phrases. Weak chromatic polynomial, mixed graph, poset, $\omega$-labeling, order polynomial, combinatorial reciprocity theorem.

We thank Thomas Zaslavsky and two anonymous referees for various helpful suggestions about this paper, and Ricardo Cortez and the staff at MSRI for creating an ideal research environment at MSRI-UP. This research was partially supported by the NSF through the grants DMS-1162638 (Beck), DMS-0946431 (Young), and DMS-1156499 (MSRI-UP REU), and by the NSA through grant H98230-11-1-0213. 
a combinatorial reciprocity theorem. More recently, Beck, Bogart, and Pham proved the following analogue of Stanley's reciprocity theorem for the strong chromatic polynomial of a mixed graph [1]:

Theorem 1. For any mixed graph $G=(V, E, A)$ and positive integer $k,(-1)^{|V|} \widehat{\chi}_{G}(-k)$ equals the number of $k$-colorings of $G$, each counted with multiplicity equal to the number of compatible acyclic orientations of $G$.

In this paper, we complete the picture by proving a reciprocity theorem for weak chromatic polynomials $\chi_{G}(k)$ of mixed graphs. A coloring $c$ and an orientation of $G$ are intercompatible if for every $u \rightarrow v$ in the orientation,

$$
c(u) \leq c(v) \text { if } u v \in E(G) \quad \text { and } \quad c(u)<c(v) \text { if } \overrightarrow{u v} \in A(G) .
$$

Our main results is:

Theorem 2. For any acyclic mixed graph $G=(V, E, A)$ and positive integer $k,(-1)^{|V|} \chi_{G}(-k)$ equals the number of $k$-colorings of $G$, each counted with multiplicity equal to the number of intercompatible acyclic orientations of $G$.

One can prove this theorem along somewhat similar lines to the (geometric) approach used in [1], though there are subtle details that distinguish the case of weak chromatic polynomials from the one of strong chromatic polynomials. For example, although both Theorems 1 and 2 result in relating $k$-colorings of a mixed graph to its acyclic orientations, the reciprocity theorem for strong chromatic polynomials applies to all mixed graphs $G$, while the reciprocity theorem for weak chromatic polynomials requires the condition that $G$ be an acyclic mixed graph: without this condition, Theorem 2 is not true.

Our proof of Theorem 2 applies Stanley's reciprocity theorem for order polynomials, stated in Section 2, which also contains the proof of Theorem 2. In Section 3 we give a deletion-contraction method for computing the weak and strong chromatic polynomials for mixed graphs, as well as an example that shows Theorem 2 may not hold for mixed graphs that are not acyclic.

\section{Posets, Order Polynomials, and the Proof of Theorem 2}

Recall that a partially ordered set (a poset) is a set $P$ with a relation $\preceq$ that is reflexive, antisymmetric, and transitive. Following [7] (see also [9, Chapter 3]), we define an $\omega$-labeling of a poset with $n$ elements as a bijection $\omega: P \rightarrow[n]$, and the order polynomial $\Omega_{P, \omega}(k)$ as

$$
\Omega_{P, \omega}(k):=\#\left\{\left(x_{1}, x_{2}, \ldots, x_{n}\right) \in[k]^{n}: \begin{array}{l}
x_{u} \leq x_{v} \text { if } u \preceq v \text { and } \omega(u)<\omega(v) \\
x_{u}<x_{v} \text { if } u \preceq v \text { and } \omega(u)>\omega(v)
\end{array}\right\} .
$$

(Here \#S denotes the cardinality of the set $S$.) Stanley [7] proved that $\Omega_{P, \omega}(k)$ is indeed a polynomial in $k$. The complementary labeling to $\omega$ is the $\bar{\omega}$-labeling of $P$ defined by $\bar{\omega}(v):=n+1-\omega(v)$. Thus

$$
\Omega_{P, \bar{\omega}}(k)=\#\left\{\left(x_{1}, x_{2}, \ldots, x_{n}\right) \in[k]^{n}: \begin{array}{l}
x_{u}<x_{v} \text { if } u \preceq v \text { and } \omega(u)<\omega(v) \\
x_{u} \leq x_{v} \text { if } u \preceq v \text { and } \omega(u)>\omega(v)
\end{array}\right\} .
$$

Theorem 3 (Stanley [7]). $\Omega_{P, \omega}(-k)=(-1)^{|P|} \Omega_{P, \bar{\omega}}(k)$.

The reciprocity relation given in Theorem 3 takes on a special form when $\omega$ is a natural labeling of $P$, i.e., one that respects the order of $P$. (It is easy to see that every poset has a natural labeling.) In this case $\Omega_{P, \omega}(k)$ simply counts all order preserving maps $x: P \rightarrow[k]$ (i.e., $u \preceq v \Longrightarrow x_{u} \leq x_{v}$ ), whereas $\Omega_{P, \bar{\omega}}(k)$ couns all strictly order preserving maps $x: P \rightarrow[k]$ (i.e., $u \prec v \Longrightarrow x_{u}<x_{v}$ ). Theorem 3 implies that these two counting functions are reciprocal. 
For a mixed graph $G=(V, E, A)$ with $n$ vertices, the weak chromatic polynomial $\chi_{G}(k)$ can be written as

$$
\chi_{G}(k)=\#\left\{\left(x_{1}, x_{2}, \ldots, x_{n}\right) \in[k]^{n}: \begin{array}{l}
x_{u} \leq x_{v} \text { if } \overrightarrow{u v} \in A \\
x_{u} \neq x_{v} \text { if } u v \in E
\end{array}\right\} .
$$

Each acyclic orientation of $G$ can be translated into a poset by letting $P=V(G)$ and introducing, for each $u \rightarrow v$ in the orientation, the relation $u \preceq v$.

Throughout the remainder of this section, we fix an acyclic mixed graph $G$ and denote by $G_{1}, G_{2}, \ldots, G_{m}$ the (acyclic) orientations of $G$. For each $1 \leq i \leq m$, denote $P_{i}$ as the poset created by the orientation $G_{i}$, and let $\phi_{G_{i}}(k)$ be the number of weak proper $k$-colorings of $G_{i}$ that are also weak proper $k$-colorings of $G$.

Lemma 4. If $G$ is an acyclic mixed graph, then $\chi_{G}(k)=\sum_{i=1}^{m} \phi_{G_{i}}(k)$.

Proof. It is clear that each weak proper $k$-coloring of $G$ is a weak proper $k$-coloring of $G_{i}$ for some $1 \leq i \leq m$. Conversely, assuming $E(G) \neq \varnothing$, for any $1 \leq i<j \leq k$, there is some $u v \in E(G)$ such that $u \rightarrow v$ in $G_{i}$ and $v \rightarrow u$ in $G_{j}$. This implies that there is no weak proper coloring that is a weak proper $k$-coloring of $G_{i}$ and $G_{j}$. If $E(G)=\varnothing$ then $G$ is the only orientation of itself.

Lemma 5. For each $G_{i}$, there exists an $\omega_{i}$-labeling of $P_{i}$ such that

$$
\phi_{G_{i}}(k)=\Omega_{P_{i}, \omega_{i}}(k) \text {. }
$$

Moreover, $\Omega_{P_{i}, \bar{\omega}_{i}}(k)$ is the number of $k$-colorings intercompatible with $G_{i}$.

Proof. Given the orientation $G_{i}$, let $R_{i}$ be the orientation of $G$ obtained by reversing the orientation of the edges in $G_{i}$ (but not the arcs). We will construct $\omega_{i}$ recursively.

Since $R_{i}$ is acyclic, there exists a vertex $v \in V$ such that all edges and arcs incident to $v$ are oriented away from it. Set $\omega_{i}(v):=1$ and remove $v$ and the arcs incident to $v$. Since $R_{i}$ is acyclic, $R_{i}-v$ must also be acyclic. Now repeat, assigning each vertex in the process consecutive $\omega_{i}$-labels. This gives $\omega_{i}$-labels that satisfy

$$
u \rightarrow v \text { in } R_{i} \quad \Longrightarrow \quad \omega_{i}(u)<\omega_{i}(v),
$$

resulting in an $\omega_{i}$-labeling of $P_{i}$, the poset corresponding to $G_{i}$, that satisfies for $u \preceq v$

$$
\begin{array}{lll}
\omega_{i}(u)<\omega_{i}(v) & \Longrightarrow & \overrightarrow{u v} \in A(G), \\
\omega_{i}(u)>\omega_{i}(v) & \Longrightarrow & u v \in E(G) .
\end{array}
$$

So

$$
\begin{aligned}
\Omega_{P_{i}, \omega_{i}}(k) & =\#\left\{\left(x_{1}, x_{2}, \ldots, x_{n}\right) \in[k]^{n}: \begin{array}{l}
x_{u} \leq x_{v} \text { if } u \preceq v \text { and } \omega_{i}(u)<\omega_{i}(v) \\
x_{u}<x_{v} \text { if } u \preceq v \text { and } \omega_{i}(u)>\omega_{i}(v)
\end{array}\right\} \\
& =\#\left\{\left(x_{1}, x_{2}, \ldots, x_{n}\right) \in[k]^{n}: \begin{array}{l}
x_{u} \leq x_{v} \text { if } u \rightarrow v \text { in } G_{i} \text { and } \overrightarrow{u v} \in A(G) \\
x_{u}<x_{v} \text { if } u \rightarrow v \text { in } G_{i} \text { and } u v \in E(G)
\end{array}\right\} \\
& =\phi_{G_{i}}(k) .
\end{aligned}
$$

For the second part of the proof, recall that

$$
\begin{aligned}
\Omega_{P_{i}, \omega_{i}}(k) & =\#\left\{\left(x_{1}, x_{2}, \ldots, x_{n}\right) \in[k]^{n}: \begin{array}{l}
x_{u}<x_{v} \text { if } u \preceq v \text { and } \omega_{i}(u)<\omega_{i}(v) \\
x_{u} \leq x_{v} \text { if } u \preceq v \text { and } \omega_{i}(u)>\omega_{i}(v)
\end{array}\right\} \\
& =\#\left\{\left(x_{1}, x_{2}, \ldots, x_{n}\right) \in[k]^{n}: \begin{array}{l}
x_{u}<x_{v} \text { if } u \preceq v \text { and } \overrightarrow{u v} \in A(G) \\
x_{u} \leq x_{v} \text { if } u \preceq v \text { and } u v \in E(G)
\end{array}\right\} \\
& =\# \text { colorings intercompatible with } G_{i} .
\end{aligned}
$$


Proof of Theorem 2. If $G$ is an acyclic mixed graph, then by Lemma 4 .

$$
\begin{aligned}
\chi_{G}(-k) & =\sum_{i=1}^{m} \phi_{G_{i}}(-k) \\
& =\sum_{i=1}^{m} \Omega_{P_{i}, \omega_{i}}(-k) \quad \text { by Lemma 5) } \\
& \left.=\sum_{i=1}^{m}(-1)^{\left|P_{i}\right|} \Omega_{P_{i}, \overline{\omega_{i}}}(k) \quad \text { by Theorem } 3\right) \\
& =(-1)^{|V|} \sum_{i=1}^{m} \Omega_{P_{i}, \overline{\omega_{i}}}(k) .
\end{aligned}
$$

By applying Lemma 5 again, the proof is completed.

\section{Deletion-Contraction Computations}

Let $G=(V, E, A)$ be a mixed graph, $e \in E(G)$, and $a \in A(G)$. Define $G-e=(V, E-e, A)$ as the mixed graph with edge $e$ deleted and $G-a=(V, E, A-a)$ as the mixed graph with arc $a$ deleted. An edge or arc is contracted by deleting the edge or arc and identifying the vertices incident to it (keeping only one copy of each edge and arc). Denote $G / e$ as the mixed graph obtained by contracting edge $e$ in $G$ and $G / a$ as the mixed graph obtained by contracting arc $a$ in $G$. The standard proof for the deletion-contraction formula for (unmixed) graphs gives:

Proposition 6. If $G$ is a mixed graph and $e \in E(G)$, then

$$
\chi_{G}(k)=\chi_{G-e}(k)-\chi_{G / e}(k) .
$$

Define $G_{a}$ as the mixed graph $G$ with arc $a$ directed in the reverse direction. In other words, if $a=\overrightarrow{u v}$ then $G_{a}=(V, E, A-\{\overrightarrow{u v}\} \cup\{\overrightarrow{v u}\})$.

Proposition 7. If $G$ is a mixed graph and $a \in A(G)$, then

$$
\chi_{G}(k)+\chi_{G_{a}}(k)=\chi_{G-a}(k)+\chi_{G / a}(k) .
$$

Proof. Let $a=\overrightarrow{u v}, C$ be the set of weak proper $k$-colorings of $G$, and $C_{a}$ be the set of weak proper $k$-colorings of $G_{a}$. Therefore, $\chi_{G}(k)+\chi_{G_{a}}(k)=\left|C \cup C_{a}\right|+\left|C \cap C_{a}\right|$.

A coloring $c \in C \cup C_{a}$ if and only if $c$ is a weak proper $k$-coloring of $G-a$. A coloring $c \in C \cap C_{a}$ if and only if $c(u)=c(v)$ and $c$ corresponds to a weak proper $k$-coloring of $G / a$ in which the vertex created by identifying $u$ and $v$ is colored with $c(u)$. Therefore, $\chi_{G-a}(k)=\left|C \cup C_{a}\right|$ and $\chi_{G / a}(k)=\left|C \cap C_{a}\right|$.

Propositions 6 and 7 give the following equations:

$$
\begin{aligned}
& \chi_{G}(k)=\chi_{G-e}(k)-\chi_{G / e}(k), \\
& \chi_{G}(k)=\chi_{G-a}(k)+\chi_{G / a}(k)-\chi_{G_{a}}(k) .
\end{aligned}
$$

Equation (1) is very useful in computing the weak chromatic polynomials since it recursively gives the weak chromatic polynomial of a mixed graph as a difference of (in the number of vertices or edges) smaller mixed graphs. On the other hand, equation (2) gives the weak chromatic polynomial of $G$ in terms of $G_{a}$, which is not a smaller graph. However, we will show how it can be used in computation.

A directed graph $G=(V, \varnothing, A)$ is strongly connected if for any pair of vertices $u, v \in V$ there exists a directed path from $u$ to $v$. 
Proposition 8. If $G$ is a strongly connected directed graph, then $\chi_{G}(k)=k$.

Proof. Fix $u \in V$, and let $c$ be a weak proper coloring of $G$. Since there is a directed path from $u$ to any $v$ and vice versa, $c(v) \leq c(u) \leq c(v)$ for every $v \in V$. Therefore, $c(u)=c(v)$ for every $v \in V$, and since there are $k$ colors that can be assigned to $u, \chi_{G}(k)=k$.

Given a subgraph $S$ of $G$, denote $G / S$ as the mixed graph $G$ with all edges and arcs of $S$ removed and all vertices of $S$ identified to one vertex; resulting parallel edges/arcs should be replaced by a single edge/arc.

Proposition 9. Let $G$ be a mixed graph and $S$ be a strongly connected directed subgraph of $G$. Then $\chi_{G}(k)=\chi_{G / S}(k)$.

Proof. Let $s$ be the vertex that $S$ contracts to in $G / S$. For each weak proper $k$-coloring of $G$, the vertices of $S$ must all be colored the same color $j$. By defining $c(s)=j$ we get a bijection between the weak proper $k$-colorings of $G$ and $G / S$.

Computing the weak chromatic polynomial of a mixed graph is reduced to computing the weak chromatic polynomial of smaller directed graphs by applying Proposition 6. Computing the weak chromatic polynomial of a directed graph is reduced to computing the weak chromatic polynomial of smaller acyclic directed graphs (directed trees) by recursively reversing arcs and applying Proposition 7 until a strongly connected subgraph is created and Proposition 9 can be applied. Note that a strongly connected subgraph of a directed graph can be obtained by reversing arcs as long as the underlying graph is not acyclic.

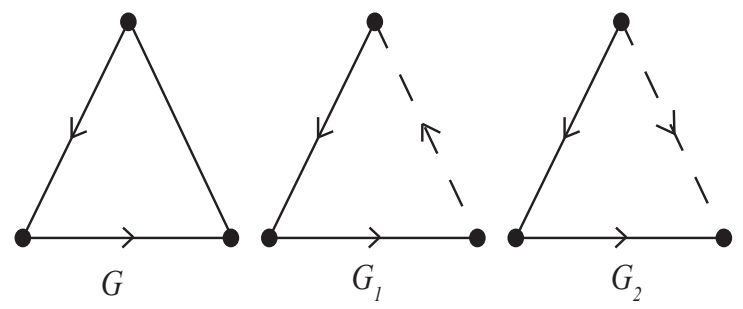

FiguRE 1. A mixed graph $G$ and its two orientations.

As an example, let $G=(\{u, v, w\},\{u v\},\{v \vec{w}, \overrightarrow{w u}\})$ (shown in Figure 1). $G$ is a cyclic mixed graph since it has an orientation, $G_{1}$, that contains a directed cycle. Consider $k=2$. If $c$ is an intercompatible coloring of $G_{1}$ or $G_{2}$, then $c(v)<c(w)<c(u)$. Therefore, $G_{1}$ and $G_{2}$ have no intercompatible colorings and the number of 2-colorings of $G$, each counted with multiplicity equal to the number of intercompatible acyclic orientations of $G$ is 0 .

We now use contraction and deletion, with $e=u v$, to compute the weak chromatic polynomial of $G$. The contracted graph $G / e$ (see Figure 2 is a strongly connected directed graph, so $\chi_{G / e}(k)=k$. In $G-e$, there are $(k-i+1) i$ weak proper $k$-colorings with $c(w)=i$. Therefore,

$$
\chi_{G-e}(k)=\sum_{i=1}^{k}(k-i+1) i=\frac{(k+2)(k+1) k}{3}
$$




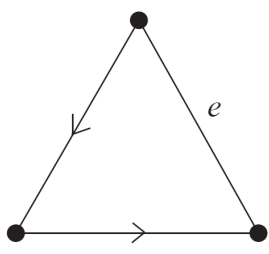

G

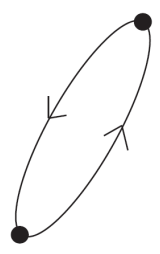

G/e

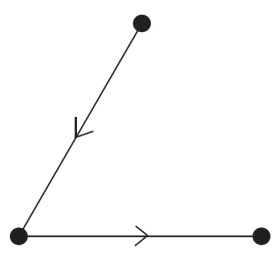

$G-e$

FiguRE 2. $G$ and its contraction and deletion.

and so $\chi_{G}(k)=\frac{1}{3}(k+2)(k+1) k-k$. We can now see that Theorem 2 does not hold for $G$ since $\chi_{G}(-2)=-2$.

\section{REFERENCES}

1. Matthias Beck, Tristram Bogart, and Tu Pham. Enumeration of Golomb rulers and acyclic orientations of mixed graphs. Electronic J. Combinatorics, 19:P42, 2012.

2. Hanna Furmańczyk, Adrian Kosowski, Bernard Ries, and Paweł Żyliński. Mixed graph edge coloring. Discrete Math., 309(12):4027-4036, 2009.

3. Pierre Hansen, Julio Kuplinsky, and Dominique de Werra. Mixed graph colorings. Math. Methods Oper. Res., 45(1):145-160, 1997.

4. Tomer Kotek, Johann A. Makowsky, and Boris Zilber. On counting generalized colorings. In Computer science logic, volume 5213 of Lecture Notes in Comput. Sci., pages 339-353. Springer, Berlin, 2008.

5. Yuri N. Sotskov and Vjacheslav S. Tanaev. Chromatic polynomial of a mixed graph. Vescī Akad. Navuk BSSR Ser. Fìz.-Mat. Navuk, (6):20-23, 140, 1976.

6. Yuri N. Sotskov, Vjacheslav S. Tanaev, and Frank Werner. Scheduling problems and mixed graph colorings. Optimization, 51(3):597-624, 2002.

7. Richard P. Stanley. Ordered structures and partitions. American Mathematical Society, Providence, R.I., 1972. Memoirs of the American Mathematical Society, No. 119.

8. Richard P. Stanley. Acyclic orientations of graphs. Discrete Math., 5:171-178, 1973.

9. Richard P. Stanley. Enumerative Combinatorics. Volume 1, volume 49 of Cambridge Studies in Advanced Mathematics. Cambridge University Press, Cambridge, second edition, 2012.

Department of Mathematics, San Francisco State University, San Francisco, CA 94132, USA

E-mail address: mattbeck@sfsu.edu

Department of Computing and Mathematical Sciences, California Institute of Technology, Pasadena CA 91125, USA

E-mail address: blado@caltech.edu

Department of Mathematics, Morehouse College, Atlanta GA 30314, USA

E-mail address: josephdcrawford@yahoo.com

Department of Mathematics, Amherst College, Amherst MA 01002, USA

E-mail address: tjeanlouis13@amherst.edu

Department of Mathematics, Iowa State University, Ames IA 50011, USA

E-mail address: myoung@iastate.edu 